
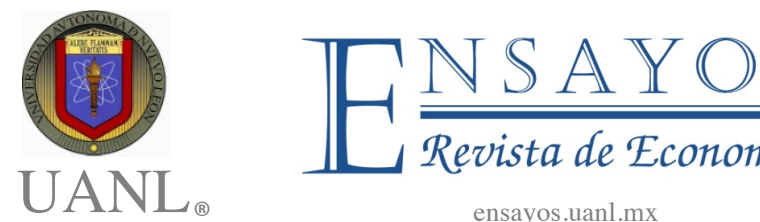

Revista de Economía

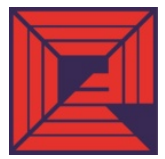

\title{
Desigualdad salarial entre trabajadores con y sin discapacidad en México, ¿Discriminación o menor productividad?
}

\section{Salary Inequality Among Workers with and without Disabilities in Mexico, Discrimination or Lower Productivity?}

\section{Reyna Elizabeth Rodríguez Pérez Felipe de Jesús García Alvarado**}

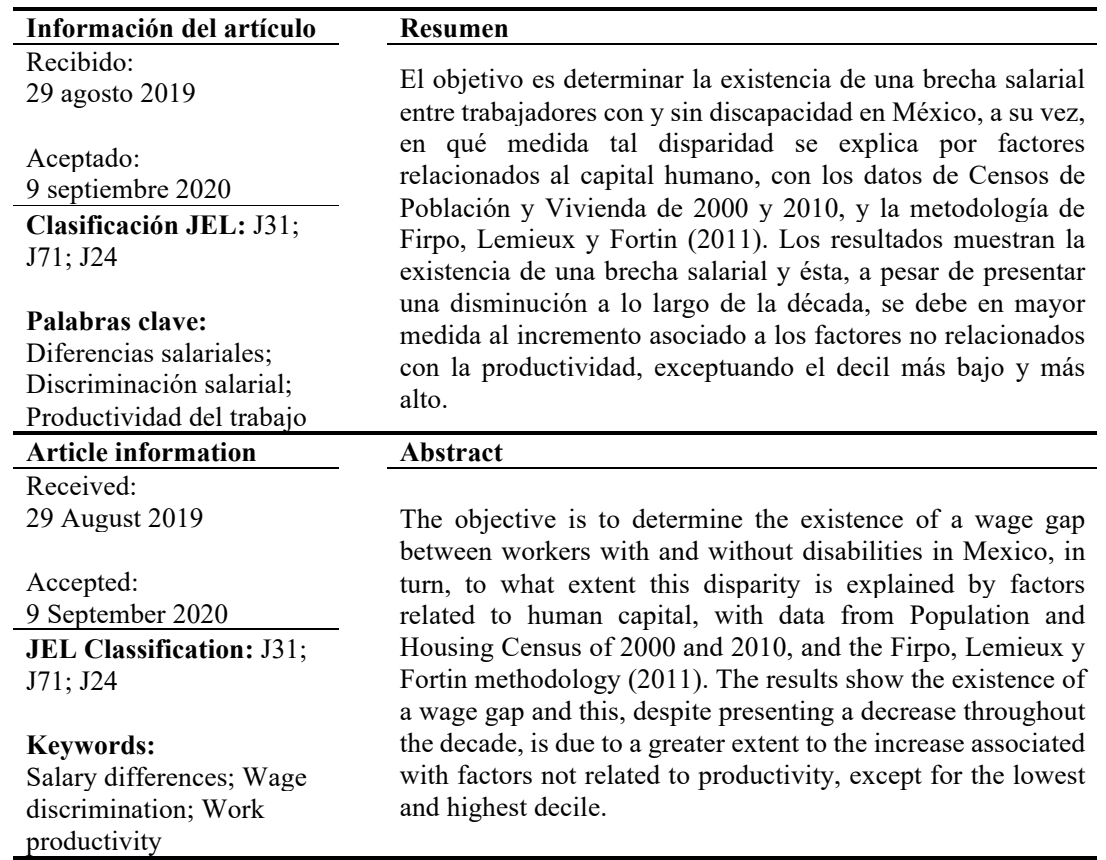

\footnotetext{
*Universidad Autónoma de Coahuila, reynarodriguez@uadec.edu.mx; ** Universidad Autónoma de Coahuila

ISSN Electrónico: 2448-8402 | ISSN Impreso: 1870-221X | (C2020 Los autores @) () 


\section{Introducción}

Es reconocido por la Organización de las Naciones Unidas, la "minoría más amplia del mundo" (ONU, 2008) es constituida por las personas con discapacidad (PCD). Según el Informe mundial sobre discapacidad, publicado por la Organización Mundial de la Salud (OMS) y el Banco Mundial (BM) en el 2011 "más de 1,000 millones de personas viven con algún tipo de discapacidad" (OMS y BM, 2011). Los organismos internacionales reconocen que este colectivo no tiene las mismas oportunidades de acceso a la recreación, los servicios de salud, educación, trabajo, etc., en comparación de la población sin discapacidad (PSD), y a su vez enfrentan barreras físicas, sociales y económicas derivadas de la discriminación y la errónea creencia colectiva de su inferioridad.

En lo que respecta a las brechas salariales por motivo de discapacidad, diversos estudios a nivel internacional han encontrado que, en efecto, existen diferencias significativas en los ingresos de las personas con y sin discapacidad. Por ejemplo, Kidd et al. (2000) evidencian para Reino Unido una brecha salarial del $14 \%$, en donde el $50 \%$ de la brecha se debe a factores no explicados por productividad; Skogman (2004), encuentra para Suecia una discriminación salarial del 50 y $60 \%$; Barletti e Yllescas (2014) encuentran para Perú una diferencia significativa del $17 \%$, en donde el $33.46 \%$ de la brecha se debe a factores no explicados por las características; Halima y Rococo (2014) estiman la brecha para Francia en 14.3\%, con un porcentaje de discriminación asociado del 50\%.; Pagán y Marchante (2004) en España sitúan la diferencia salarial en $16.6 \%$, con un porcentaje no explicado de la brecha del 42.98\%; Malo y Pagan (2012) encuentra una discriminación superior al 50\% para una muestra de once países de Europa; Benito et al (2016) encuentra un $40 \%$ de la brecha salarial que sufren las personas con este tipo de discapacidad y el $60 \%$, corresponde a discriminación para el caso de Estados Unidos.

Por su parte, para el caso mexicano, se encontraron únicamente un par de investigaciones que logran evidenciar la desigualdad y discriminación salarial hacia el colectivo de personas con discapacidad. Un primer documento es el elaborado por Márquez y Reyes (2015), en donde encuentran que las personas sin discapacidad obtienen un salario equivalente a 1.7 veces el salario de los individuos con discapacidad, y donde el $83 \%$ de la brecha se debe a factores no asociados con las características observadas. Finalmente, Castro, Rodríguez y Galván (2017) realizan una investigación para el caso de Coahuila de Zaragoza, en donde muestran la existencia de la brecha salarial entre colectivos, de 0.26 puntos logarítmicos en el 2000 , y de 0.18 en el 2010 , y una posible discriminación asociada de $90 \%$ en el 2000, y 60\% en el 2010 . 
En todas estas investigaciones el factor no explicado (aspectos atribuidos a discriminación o no explicado por las características de capital humano) contribuye en gran medida al diferencial salarial que existe entre estos grupos de personas, aunque varía por país y por el tipo de metodología empleada, este elemento siempre está presente.

Una vez descrito que es una realidad latente la desigualdad y discriminación salarial contra las personas con discapacidad en México y el mundo, conviene realizarse algunas preguntas de investigación: ¿Qué tan fuerte es la brecha salarial entre las personas con y sin discapacidad en México? Si en efecto, existió una brecha salarial por razones de discapacidad en ambos años, ¿Habrá algún cambio significativo en la brecha salarial de estos dos grupos entre 2000 y 2010? ¿Qué porcentaje de la brecha se explica por factores relacionados con la productividad del individuo? y ¿qué porcentaje es atribuido a factores no explicados?

A partir de las preguntas de investigación, el objetivo de este trabajo se centra en determinar la existencia de una brecha salarial significativa entre los trabajadores con discapacidad y sin discapacidad para México, a su vez, en qué medida tal disparidad se explica por factores relacionados al capital humano, y qué cantidad no lo hace.

A partir de lo anterior, la hipótesis de esta investigación se establece de la siguiente manera: En ambos años, 2000 y 2010, existe desigualdad salarial entre personas con y sin discapacidad, y a lo largo de la década, sobre la brecha existente en los extremos de la distribución salarial, tienen mayor participación los factores ajenos a la productividad.

La metodología empleada para la realización de este documento se enfoca en el modelo de descomposición decílico propuesto por Fortin, Lemieux y Firpo (FLF, 2011), el cual, a la par de otorgar los valores explicados y no explicados de la brecha salarial, indica el aporte que tienen las características observadas en la fracción explicada de la brecha. Lo anterior utilizando los microdatos de los Censos Generales de Población y Vivienda de los años 2000 y 2010 (cuestionarios ampliados). La importancia que tiene realizar el estudio a lo largo de la distribución salarial es debido a que los niveles de salario no son homogéneos, y tomar valores promedio generaría sesgos en la estimación de la desigualdad y la discriminación salarial entre ambos grupos, personas con discapacodad y personas sin discapcidad,. A su vez, resulta relevante identificar si la discriminación es más profunda en la cola izquierda, o en la cola derecha de la distribución. Es decir, establecer comparaciones relativas entre los individuos con y sin discapacidad que obtienen las menores y mayores retribuciones salariales. 
Los resultados más relevantes se pueden resumir en los siguientes puntos: i) en efecto, existe desigualdad salarial hacia los trabajadores con discapacidad, la cual se redujo entre 20.8 a $48.7 \%$ en la década $2000-2010$; ii) los porcentajes de la brecha asociados a factores explicados por las características fluctuaron entre $27.7 \%$ (decil más alto) a 55.6\% (decil más bajo) para el 2000, y 19.54.1\% (decil 75) a 62.89 (decil más bajo) para el año 2010; iii) de tales porcentajes asociados a características observadas, fueron las variables de tipo personal, en específico, las referidas a la educación, las que tuvieron mayor participación dentro de la sección explicada; iv) los porcentajes asociados a los factores no observados (comúnmente conocidos como discriminación) se ubicaron entre 44.4 a $72.3 \%$ en el 2000 , y 37.1 a $80.5 \%$ en el 2010 ; y v) en la década de referencia, la discriminación creció en los segmentos intermedios de nivele de ingreso (deciles 25, 50 y 75), mientras que presentó reducción en los extremos de la distribución (decil 10 y 90).

El presente documento se inicia, en su primer apartado, con una revisión teórica sobre la desigualdad, la discriminación salarial y se enuncian los principales enfoques explicativos de estos fenómenos. Posteriormente se presenta una revisión de la evidencia empírica a nivel internacional y nacional. En el segundo apartado, se detalla la metodología, los datos y las variables cualitativas y cuantitativas utilizadas en el modelo estimado. En un tercer apartado se presenta con detalle los resultados de la estadística descriptiva y el modelo de descomposición. Y finalmente, se presentan las conclusiones generales derivadas de los resultados y su contraste con la evidencia empírica.

\section{Revisión de literatura y evidencia empiríca 1.1 Concepto y tipos de discapacidades}

Según la Clasificación Internacional del Funcionamiento, de la Discapacidad $y$ de la Salud (CIF) publicado por la Organización Mundial de la Salud, "discapacidad es un término genérico que incluye déficits [deficiencias], limitaciones en la actividad y restricciones en la participación. El término indica aspectos negativos de la interacción entre un individuo y sus factores contextuales" (OMS, 2001, pág. 206).

A partir de esta definición es preciso detallar los aspectos mencionados en ella. En primer lugar, las deficiencias constituyen la "anormalidad o pérdida de una estructura corporal o de una función fisiológica (física o mental)" (OMS, 2001, pág. 207). Es decir, que algún órgano o parte del cuerpo manifieste una desviación significativa respecto a la norma estadística establecida en una población. En segundo lugar, las limitaciones a la actividad son "las dificultades que un individuo puede tener para realizar sus actividades" (OMS, 2001, pág. 207). Por último, las restricciones en la participación "son los problemas que puede experimentar un individuo para implicarse en situaciones 
vitales... en una cultura o sociedad" (OMS, 2001, pág. 207). Es decir, la segregación de la que puede ser víctima en la interacción con los demás miembros de un colectivo.

Las discapacidades pueden ser múltiples y muy variadas, sin embargo, recogen suficiente información susceptible de ser analizada desde diferentes campos de estudio al relacionarlas con otras variables de tipo económico, social, demográfico, entre otras, información que de igual manera se recopila en los censos u otro tipo de instrumentos. Por ejemplo, Malo (2007), menciona que la antigua política social distinguía entre pobres "validos" y pobres "invalidos". Los primeros eran capaces de trabajar y, por tanto, lo que se suponía que habia que hacer con ellos era ayudarles a encontrar un trabajo. Los segundos serían incapaces de trabajar y, se les tenía que asignar una pensión o algun tipo de ayuda, por lo que este grupo es necesariamente el de las personas con discapacidad. Por lo tanto, desde esta perspectiva se puede deducir, que si puede participar activamente en el mercado laboral, entonces no es una persona con discapacidad. Esto resulta ser un problema, ya que la mayor parte de las encuestas o datos administrativos que emiten distintos países, definen la discapacidad como una limitante para trabajar, por lo que analizar el mercado laboral de las personas con discapacidad resulta un reto importante.

\subsection{Desigualdad y discriminación salarial}

Una de las vertientes de análisis de la economía laboral, es el estudio de las desigualdades sociales entre grupos de población. La desigualdad salarial puede definirse como la diferencia existente entre los salarios percibidos por los trabajadores de dos grupos, calculada sobre la base de la diferencia media entre los ingresos brutos por hora de todos los trabajadores (CE, 2014). Es necesario recalcar que salario implica la parte exclusiva a la remuneración que una persona obtiene por el uso que una empresa u organización hace de sus servicios productivos. Es decir, la remuneración que obtiene por la venta de su fuerza de trabajo.

Desde el campo de las ciencias sociales, a la par del desarrollo del instrumental analítico que busca probar la desigualdad en salarios, también han surgido múltiples enfoques teóricos acerca de las posibles causas que explican las desigualdades en la remuneración de los individuos.

En materia económica se habla de discriminación laboral "cuando, dos trabajadores con la misma capacidad productiva pero que difieren en alguna característica personal no relacionada con ésta, uno recibe un trato inferior en cuanto a posibilidades de obtener empleo y en las condiciones de trabajo" (Guataquí, Baquero, y Sarmiento, 2000, pág. 3). En el contexto de este documento, el trato hacia las personas con discapacidad no es equitativo 
respecto a las personas sin discapacidad. Con esta definición, Guataquí et al. (2000) logran asociar cuatro tipos de discriminación que ocurren durante la obtención y permanencia en un empleo: discriminación salarial, en el empleo, ocupacional y en la adquisición de capital humano.

Aunado a lo anterior, la discriminación salarial se constituye cuando en condiciones iguales de trabajo, experiencia, educación y demás factores observables relacionados directamente con la productividad, un individuo recibe menor remuneración por el uso de su fuerza de trabajo $\mathrm{y}$, en consecuencia, se manifiesta la discriminación salarial por juicios subjetivos de los empleadores a causa de uno o varios tipos de discriminación antes mencionados. Cabe resaltar que "no necesariamente toda diferencia salarial es el resultado de discriminación, ni toda práctica discriminatoria resulta en diferencias salariales” (Tenjo, Ribero y Bernat, 2005, pág. 8).

La discriminación en el mercado laboral hacia las personas con discapacidad se manifiesta de diferentes formas, es por eso que en el mundo académico se ha intentado explicar este fenómeno con el desarrollo de algunos modelos. Y aunque las teorías de la discriminación en el mercado laboral se centran principalmente en modelos de discriminación por género, estos pueden ser fácilmente adaptados a la discriminación por motivos de discapacidad (personas con y sin discapacidad), ya que, en la población en general estos grupos se consideran mutuamente excluyentes. En este contexto, Lago (2002) divide en cinco grupos tales modelos: teoría del gusto por la discriminación, discriminación estadística, modelo "monopolístico" de discriminación, el modelo overcowding de discriminación y modelos institucionales de discriminación. Sin embargo, los más utilizados en el estudio de este fenomeno son: la teoría del gusto por la discriminación, la discriminación estadística y el modelo overcowding.

Con respecto al primer modelo, Becker (1957) argumenta que la discriminación supone la existencia de un grupo mayoritario y otro minoritario, en este caso el que tiene algun tipo de discapacidad. Este último grupo recibe un trato inferior (un salario menor), con relación al grupo mayoritario. El origen de esta discriminación esta basado en el gusto por los individuos y se define en las actividades económicas como la renuncia voluntaria a utilidades e ingresos para satisfacer prejuicios. La discriminación supone un costo o una perdida de la eficiencia productiva de la empresa. El modelo supone que el agente discriminador es el empleador, pero también puede adquirir tal condición otros dos grupos, como los compañeros de trabajo y los clientes.

Por su parte, el modelo de discriminación estadística porpuesto por Phelps (1972) y Aigner y Cain (1977), argumentan que la discriminación no se produce como un prejuicio personal, sino porque se presume diferencias de 
productividad significativas entre trabajadores. En este modelo, los empleadores realizan la contratación de los trabajadores en situaciones de información imperfecta, utilizando criterios para evaluar a los candidatos sobre las características de productividad media de las personas. Puesto que las evaluaciones de las que disponen los empresarios son sesgadas, se produce un nuevo fenomeno de la discriminación por condición de discapacidad.

Finalmente, el modelo overcrowding propuesto por Berman (1974), analiza las diferencias salariales por sexo, pero si trasladamos el mismo enfoque hacia las personas con o sin discapacidad, se podría suponer que la segregación en el empleo se puede dar de manera horizontal o vertical. Si se produce horizontalmente, se observa que las personas sin discapacidad ofrecen su trabajo en sectores concretos de actividad y las personas con discapacidad estan empleados mayoritariamente en otros.

Por su parte, Yuker (1988) menciona que es necesario realizar investigaciones que consideren el tipo de discapacidad que se estudia (utilizando diferentes definiciones, e incluyendo niveles de severidad), y que las discapacidades se clasifiquen en función de la aceptación social general; la voluntad de emplear, enseñar, rehabilitar o casarse; o la aceptabilidad como una discapacidad personal. De igual manera, agrega que la descripción de la discapacidad también podría incluir información sobre las habilidades y competencias de cada persona, por ejemplo, su disposición a contratar a un trabajador con esa discapacidad, siempre y cuando la persona tenga todas las habilidades y competencias que requiere el trabajo.

Con lo anterior se deduce que, si la discriminación tiene su origen en prejuicios, la sociedad se beneficia de su eliminación. Según Becker (1957), desde la perspectiva económica, la eliminación de la discriminación es eficiente, pues es un costo que no reporta más que una satisfacción psicológica por contratar a quienes las empresas no contratarían por un salario sin discriminación. Por su parte, Malo (2017) argumenta que si la fuente de diferencia es la discriminación estadística, la cuestión no es tan sencilla. Dado que se usa la variable de discapacidad para resolver un problema de información (no se sabe la productividad individual, sino solo la del promedio del grupo de discapacitados) impedir el uso de esa variable para evitar la discriminación estadística, conduce a una disminución de la eficiencia. En cierto sentido, la discriminación es el precio que se paga por la existencia de información imperfecta. Por tanto, si la fuente de las diferencias salariales discriminatorias presenta este tipo de problemas de información en el mercado de trabajo, la estrategia consistiría en aumentar y mejorar la información sobre las personas con discapacidad con la que cuentan las empresas a la hora de contratar. El desarrollo de servicios de intermediación laboral especializados (sobre todo, por tipo de discapacidad o nivel de severidad) puede desempeñar 
un importante papel, disminuyendo esta clase de problemas de información y colaborando así a que el empleo de las personas con discapacidad no se vea perjudicado por esta vía.

A pesar de la existencia de explicaciones teóricas de discriminación, en la realidad, las condiciones para personas con discapacidad son aún muy distintas a las de personas sin discapacidad ya que el acceso a servicios y a la igualdad de derechos y oportunidades es un reto vigente. Finalmente, la comprobación empírica de las teorías se convierte en una tarea difícil de realizar, puesto que, se desconocen las posturas y preferencias de los empleadores. Además, este tipo de investigaciones son de carácter cualitativo.

\subsection{Evidencia empírica de la desigualdad y discriminación salarial contra las personas con discapacidad}

Los documentos presentados en esta sección se centran casi por completo en los estudios que han realizado economistas a nivel internacional (Reino Unido, Italia, Perú, Francia, España, la región de Andalucía en España, once países de la Unión Europea, Estados Unidos y Tamil Nadu en India), pues la literatura existente para el caso mexicano es muy escasa, de esta última cabe destacar los trabajos realizados por Márquez y Reyes (2015) y Castro, Rodríguez y Galván (2017).

A nivel internacional, utilizando la Encuesta de la Fuerza de Trabajo Británica, Kidd et al (2000), evidencian por medio de la metodología Baldwin y Johnson (1994), que existe diferencias sustanciales en los salarios y en la tasa de participación en el mercado laboral entre los trabajadores británicos que poseen alguna discapacidad y los que no. El resultado obtenido fue que existe una brecha de aproximadamente el $14 \%$ entre ambos, en donde los factores observados representaron el $50 \%$ de la disparidad, que es lo que en forma habitual se denonina discriminación salarial.

Por su parte, Skogman (2004) en un estudio para Suecia y con la aplicación de la metodología Oaxaca-Blinder (1973), muestran que existe una mayor proporción de personas con discapacidad en ocupaciones no calificadas. Este autor encuentra que las características explican la diferencia salarial en 1981, pero solo entre el $40 \%$ y el $50 \%$ en 1991, por lo que la discriminación salarial por discapacidad se ubica entre el 50 y el $60 \%$.

Para el caso de Perú, Barletti e Yllescas (2014) mediante la aplicación de estimaciones paramétricas modeladas por Oaxaca y Blinder (1973) y no paramétricas propuestas por Ñopo (2004) a de la Encuesta Nacional Especializada sobre Discapacidad (ENEDIS) y la Encuesta Nacional de Hogares (ENAHO), ambas del año 2012, demuestran la existencia de una 
brecha salarial del $17.03 \%$ entre la población discapacitada y no discapacitada, asimismo, el componente no explicado que hace referencia a la existencia de discriminación se situó en el 33.46\%.

En Francia, Halima y Rococo (2014) estudian el diferencial salarial entre las personas con discapacidad y sin discapacidad a partir de la Encuesta de Salud, Cuidado y Seguros del 2010, diferenciando en el campo de las personas con discapacidad a los que se autoevalúan con salud mala, con una enfermedad crónica o con una limitación en la actividad. Al aplicar el método de descomposición desarrollado por Lanot y Walker (1998), combinado con la de Oaxaca y Blinder (1973) que incluye la corrección de sesgo de selección desarrollado por Heckman (1979), este estudio encuentra que la tasa de salario por hora entre los individuos con un estado de salud autoevaluado deficiente es en promedio $14.2 \%$ menor que entre individuos con buen estado de salud. Similarmente, la tasa para las personas que sufren de una enfermedad crónica es $6.3 \%$ menor en comparación de las personas con buena salud, mientras que para las personas con una limitación en la actividad la tasa salarial es en promedio 4.5\% menor. Asimismo en el estudio se indica que el "componente no explicado" de las desigualdades salariales es igual al 50\%.

A través de un estudio realizado por Pagán y Marchante (2004), a partir de los microdatos del Panel de Hogares de la Unión Europea (PHOGUE) para el período 1995-2000, mediante la estimación de ecuaciones de salarios para las personas con discapacidad y sin discapacidad y corregidas del sesgo de selección mediante el modelo de dos etapas de Heckman, se estimaron las diferencias salariales entre estos dos grupos en España. Como resultado de este estudio se obtuvo que las personas con discapacidad reciben un salario igual al $83.39 \%$ del salario recibido por las personas sin discapacidad. A su vez, se muestra que el $42.98 \%$ de la diferencia salarial es atribuible a las diferencias en las características no explicadas, es decir por las diferencias en los rendimientos.

Malo y Pagan (2012) con los datos del PHOGUE, analizan a un subgrupo de las personas que tienen alguna discapacidad y que no experimentan limitaciones en actividades de la vida diaria en once países europeos. Los resultados que obtienen revelan que la diferencia salarial discriminatoria por discapacidad es muy poca para este grupo. También se encuentra que las personas con discapacidad muestran un menor salario y éste se relaciona con las características asociadas con una menor productividad, la cual se encuentra en función del nivel educativo. Sin embargo, cuando analizan el grupo de personas con discapacidades que generan limitaciones en la vida diaria, los resultados revelan la existencia de una diferencia salarial negativa asociada a la discapacidad que no se explican por las características observadas que afectan a la productividad. 
Jones, Latreille y Slone (2006), utilizando datos de la Encuesta de Demografía y Salud de 2002, examinan el impacto de la discapacidad en los resultados del mercado laboral por género en Reino Unido. Sus resultados indican que existen diferencias sustanciales tanto en la probabilidad de empleo como en los niveles de ingresos, a pesar de que la Ley sobre la discriminación por motivos de discapacidad lleva varios años en vigor. Se identifica una heterogeneidad significativa dentro del grupo de discapacitados: a los que sufren formas de discapacidad de salud mental les va especialmente mal. Las descomposiciones salariales sugieren que la "penalidad" por discapacidad es mayor para las mujeres que para los hombres. Estos autores aplican la metodología de Baldwin y Johnson (1992), encontrando que los efectos en el empleo asociados a la discriminación salarial de los discapacitados son muy pequeños.

Para el caso de Estados Unidos, Baldwin (1994) utilizó la base de datos tipo panel de la Encuesta de Ingresos y Programa de Participación de 1984. La autora calcula dos estimaciones diferentes de discriminación aplicando la técnica de descomposición de Oaxaca para personas con y sin discapacidad. En sus resultados, Baldwin (1994) obtiene que los salarios promedio por hora son US $\$ 9.38$ para los hombres sin discapacidad y $\$ 8.80$ para los hombres con discapacidad; y $\$ 6.56$ para las mujeres sin discapacidad y $\$ 5.93$ para las mujeres con discapacidad. Por lo tanto, los diferenciales de salarios observados son de $\$ 0.58$ por hora entre hombres con discapacidades y no discapacitados, en donde el $137 \%$ se debe a factores no explicados; y $\$ 0.63$ por hora entre mujeres discapacitadas y no discapacitadas, donde el factor no explicado es del $62.8 \%$.

En otro estudio para Estados Unidos, Benito et al (2016) realizan un estudio para el caso de las personas con discapacidad auditiva. Con la metodología Oaxaca-Blinder (1971), los resultados que obtienen indican que el $40 \%$ de la brecha salarial que sufren las personas con este tipo de discapacidad, se puede atribuir a características como el nivel educativo, experiencia, origen étnico o estado civil, mientras que $60 \%$ restante corresponde a discriminación.

Mitra y Sambamoorthi (2008) examinan las diferencias en el empleo y los salarios de los varones con y sin discapacidad en el mercado de trabajo rural para la región de Tamil Nadu en la India. Usando los datos de la Encuesta de Discapacidad de la Aldea del 2005, realizada por el Banco Mundial, los autores utilizan una técnica de descomposición no binaria propuesta por Farlie (1999) y obtienen una diferencia de 1.11 rupias por hora de trabajo a favor de las personas con discapacidad. Sin embargo, la investigación sugiere que los salarios no son estadísticamente diferentes entre este par de grupos.

Para el caso particular de México, existen pocos documentos que hablan acerca de la desigualdad salarial contra las personas con discapacidad. Uno de éstos 
es el estudio presentado por Márquez y Reyes (2015), en el cual utilizan los microdatos de la Encuesta Nacional de Ingresos y Gastos de los Hogares (ENIGH) del año 2010 y la implementación de dos metodologías distintas: la descomposición Oaxaca-Blinder y el emparejamiento de Nopo. Bajo la argumentación de la sobrerrepresentación de las personas sin discapacidad en empleos de alta o muy alta calificación, los autores estiman los modelos tomando únicamente a la población empleada en trabajos no calificados. Con la utilización del método de descomposición Oaxaca-Blinder, sus resultados indican que las personas sin discapacidad ganan 1.7 veces más que las personas con discapacidad, donde el $83 \%$ de esta brecha podría deberse a aspectos de discriminación. De igual forma, con la utilización del segundo método, el emparejamiento de Hugo Ñopo, los autores encuentran que las personas sin discapacidad ganan el equivalente a 1.3 veces más de lo que ganan las personas con discapacidad, siendo en esta estimación que el $68.5 \%$ de esa brecha es debido a características no explicadas.

Otro de los documentos encontrados para México, en concreto para el estado de Coahuila, es el de Castro, Rodríguez y Galván (2017), en donde utilizando los datos de los Censos de población y vivienda de los años 2000 y 2010 y a través de la utilización de la metodología Oaxaca-Blinder, evidencian la disparidad salarial entre personas con y sin discapacidad con una estimación de 0.26 puntos logarítmicos en el 2000 , y de 0.18 en el 2010. Asimismo, estos autores encuentran la existencia de discriminación hacia trabajadores con discapacidad, la cual ascendió al $90 \%$ de la brecha en el año 2000 , y a $60 \%$ en el año 2010.

En los estudios presentados se utilizan diferentes metodologías y técnicas de descomposición: Oaxaca-Blinder (1973), Hugo Ñopo (2004), Lanot y Walker (1998), Baldwin y Johnson (1994), Farlie (1999). Cabe mencionar que los autores de muy diversas naciones, separados tanto en tiempo y espacio, obtuvieron resultados distintos debido a las condiciones económicas, políticas y sociales de las que parten cada una de las investigaciones. Sin embargo, en su mayoría se observa que el fenómeno de discriminación está latente en las remuneraciones de las personas con discapacidad.

En resumen, las diferentes vertientes de los estudios de desigualdad salarial que giran en torno al grupo de personas con discapacidad, algunos realizaron el análisis diferenciando por tipo de discapacidad y se dieron cuenta que no todos los tipos de discapacidad tienen el mismo efecto sobre la brecha salarial, pues son los que tienen alguna limitación mental los que con mayor frecuencia se les discrimina en el acceso y participación en un empleo. Otra vertiente de análisis es el grado de discapacidad, cuando existe alguna limitación a la actividad, cuando es una enfermedad crónica, si es permanente o solamente temporal y se concluye también que estos factores inciden sobre la brecha 
salarial, unos en mayor medida que otros. Otra forma de analizar la brecha y a este colectivo es desde la perspectiva de género al distinguir entre hombres y mujeres. En esta también se concluye que existe una doble discriminación (por discapacidad y por género), lo cual acentúa la gravedad del problema.

Este fenómeno de desigualdad es susceptible de ser analizado desde una gran variedad de perspectivas que involucran actos de discriminación, sin embargo, el aporte de esta investigación se centra en evidenciar la desigualdad y discriminación salarial entre personas con y sin discapacidad. En este sentido, este par de fenómenos son temas escasamente analizados para el caso mexicano, y es aquí donde radica la importancia de la realización de esta investigación, ya que se considera un aporte significativo al estudio de las características económicas de este colectivo. Asimismo, adquiere relevancia el análisis intertemporal y la utilización de la metodología Fortin, Lemieux y Firpo, 2011, que permite observar las disparidades a lo largo de la distribución salarial, así como evidenciar el aporte de cada una de las variables a la sección explicada de la brecha.

\section{Datos y metodología}

El análisis de la desigualdad y discriminación salarial de este estudio se basa en los datos de los individuos (microdatos) de los Censos Generales de Población y Vivienda (CGPV) de los años 2000 y 2010. El diseño muestral aplicado al CGPV (2000 y 2010) considera a los individuos con discapacidad, definidos como aquellos que tienen alguna dificultad al realizar actividades de la vida diaria como caminar o moverse, ver, escuchar, hablar, atender su cuidado personal, poner atención o aprender, y estas limitaciones pueden ser por nacimiento, enfermedad, accidente o edad avanzada. Asimismo, el término de discapacidad, también comprende las deficiencias en las estructuras y funciones del cuerpo humano, que limitan la capacidad personal para llevar a cabo tareas básicas de la vida diaria y las restricciones en la participación social que experimenta el individuo al involucrarse en situaciones del entorno donde vive.

Es importante destacar que las limitaciones que se enumeraron para la respuesta sobre discapacidad en el censo del 2000 y 2010 eran: moverse, caminar o lo hace con ayuda; usar sus brazos y manos; es sordo o usa un aparato para oír; es mudo; es ciego o solo ve sombras; tiene algun retraso o deficiencia mental; tiene otra limitación física o mental (se pedía especificar) o no tiene limitación física o mental. Las posibilidades de respuesta respecto a las causas fueron en ambos casos: porque nacío así, por una enfermedad, por un accidente, por edad avanzada o por otra causa que en el censo de 2000 se debían especificar. 
La muestra se constituye por individuos, con y sin discapacidad, con las siguientes características en común: Población ocupada: personas que se constituyen como parte de la fuerza laboral del país ( 16 a 65 años) y que tienen un empleo remunerado. Aquellos que no se encuentran activos en el mercado laboral se excluyen del análisis. La población clasificada como remunerada y asalariada se define como aquellos individuos que trabajan por cuenta ajena y trabajadores con una jornada laboral semanal mayor a 15 horas, y menor a 72 . Además, se considera el empleo no calificado, el cual es una característica de gran importancia, debido a que, similar al estudio realizado por Márquez y Reyes (2015), se busca corregir el sesgo de autoselección, pues los individuos se clasifican como imposibilitados para trabajar, o en dado caso, se ubican en empleos de baja calificación. De no considerarse esta corrección, se sobrestimaría la brecha salarial debido a la sobrerrepresentación de las personas sin discapacidad en empleos calificados, los cuales comúnmente se asocian a salarios más altos. Por último, para fines de esta investigación se consideran como empleos no calificados aquellos que para su realización no se requiere de un conocimiento científico, teórico o práctico muy especializado.

Finalmente, es necesario aclarar que no se aplica la metodología de Heckman para corregir el posible sesgo de selección, ya que para ello se utilizan los empleos no calificados como una medida previsora de tal sesgo en la muestra $\mathrm{y}$ en las estimaciones.

Continuando con la descripción de los datos utilizados para esta investigación, las variables independientes incluidas en las estimaciones se dividen en tres tipos: i) personales; ii) de tipo de contrato; y iii) de lugar de trabajo. Ademas, siguiendo la teoría del capital humano, se busca determinar la desigualdad salarial basada en factores que se relacionan con la productividad de los trabajadores, del mismo modo con la metodología adoptada, se busca estimar la brecha salarial no explicada por razones de productividad, comúnmente conocida como discriminación.

\section{Metodología Firpo, Lemieux y Fortin (2011)}

La metodología FLF (2011), permite desarrollar descomposiciones detalladas y estimar el impacto de los cambios en las dotaciones o los rendimientos de una variable explicativa concreta en los cuantiles de interés de una distribución salarial incondicional. Esta metodología se basa en la estimación de regresión donde la variable dependiente es sustituida por una trasformación de la misma, la función de influencia recentrada (RIF), para con ella, desarrollar una descomposición estándar de Oaxaca-Blinder basada en los resultados de regresión. 
La descomposición toma, por tanto, la siguiente forma:

$$
\Delta_{Q_{\theta}}=\left(\overline{X^{s d}}-\overline{X^{d}}\right) \hat{\gamma}_{Q_{\theta}}^{*}+\left\{\overline{X^{s d}}\left(\hat{\gamma}_{Q_{\theta}}^{d}-\hat{\gamma}_{Q_{\theta}}^{*}\right)+\overline{X^{d}}\left(\hat{\gamma}_{Q_{\theta}}^{*}-\hat{\gamma}_{Q_{\theta}}^{s d}\right)\right\}
$$

Donde $\Delta_{Q_{\theta}}$ es la diferencia en el cuantil $\Delta_{Q_{\theta}}$ de las distribuiciones salariales de trabajadores sin y con discapacidad; $\overline{X^{d}}$ y $\overline{X^{d}}$ son las características observadas promedio de personas con y sin discapacidad; $\hat{\gamma}_{Q_{\theta}}^{d}, \hat{\gamma}_{Q_{\theta}}^{s d}$ y $\hat{\gamma}_{Q_{\theta}}^{*}$ son los coeficientes estimados tras la regresión de la variable RIF del cuantil $Q_{\theta}$ sobre el conjunto de variables explicativas para individuos con discapacidad, sin discapacidad y el pool de ambos colectivos, respectivamente.

Al igual que otras metodologías, en ésta se distinguen dos componentes de la descomposición: el primero representa el efecto en las diferencias en el estadístico de los cambios en características (o componente "explicado") y el segundo corresponde al efecto de los coeficientes (o componente "no explicado"). Es importante destacar que mediante este procedimiento se obtiene una descomposición detallada de la aportación de cada factor explicativo individual al diferencial a explicar (distinguiendo, a su vez, entre los correspondientes efectos asociados a dotaciones y rendimientos).

Para esta investigación será utilizada la metodología FLF (2011) para estimar la desigualdad y discriminación salarial entre los trabajadores con y sin discapacidad, y determinar el grado de influencia de cada una de las variables sobre esta disparidad. Las ventajas esta metodología se pueden resumir en dos principales puntos: primero, realiza una descomposición cuantílica, lo cual permite establecer relaciones entre la desigualdad, discriminación y el nivel de ingreso de los individuos; y segundo, para la sección explicada de la brecha, determina la influencia que las características observadas aportan a la disparidad salarial, variables que pueden favorecer o disminuir la brecha. Asimismo, la metodología no ha sido aplicada para el caso mexicano, lo cual permitirá encontrar resultados interesantes.

Detallado en el apartado de datos, en este documento, se realiza una agrupación de las variables de interés de la manera siguiente:

- Variables individuales: interacción (casado-sexo), rangos de edad, nivel educativo, experiencia, experiencia al cuadrado y pertenencia étnica. Todas ellas relacionadas con las características personales de los trabajadores.

- Variables de tipo de contrato: horas de trabajo y formalidad. Estas variables otorgan información referente a las condiciones establecidas en el contrato laboral entre empleadores y asalariados.

- Variables de lugar de trabajo: tipo de localidad urbana, rama de actividad económica y nivel de bienestar de la región. Se indican aspectos relacionados con las condiciones socio-económicas en donde se desempeñan los trabajos. 
Una vez establecida la metodología y detallada la depuración que se ha hecho a las bases de datos utilizadas, en el siguiente apartado se detallan los resultados de la estadística descriptiva y la estimación del modelo de descomposición, con el fin de evidenciar la disparidad salarial y la discriminación de la que son víctimas los trabajadores con discapacidad.

\section{Resultados}

Una vez establecida la metodología y detallada la depuración que se ha hecho a las bases de datos utilizadas, en esta sección se muestran los resultados de la estadística descriptiva y la estimación del modelo de descomposición, con el fin de evidenciar la disparidad salarial y la discriminación de la que son víctimas los trabajadores con discapacidad.

\subsection{Estadística descriptiva de la muestra de estudio}

Derivado del análisis estadístico de los Censos poblacionales se obtiene que en el año 2000, la población ascendió a 95.2 millones de habitantes ( la tabla 1), de los cuales 2.2 millones $(2.33 \%)$ presentaron algún tipo de discapacidad (motriz, visual, de lenguaje, auditiva, mental o múltiple), de estos, aproximadamente 1.14 millones se encontraba en el rango de edad de 16 a 65 años. Sin embargo, es preciso destacar que no todos ellos constituyen la fuerza laboral de PCD disponible, pues a muchos de ellos se les considera "imposibilitados para trabajar", o al menos, así lo informan las personas encuestadas al momento del censo. Es por eso que en la tabla 1 se incluye la fila "población disponible para trabajar", pues se retiran del rubro anterior a todas aquellas personas jubiladas y a las que reportaron una condición de discapacidad que les imposibilita trabajar, suma que ascendió a un millón. Aquí se detecta un detalle metodológico de diseño de encuesta, ya que las personas encuestadas autoseleccionan como "imposibilitadas para trabajar" a los individuos con discapacidad que viven en el hogar, por el simple hecho de tener alguna dificultad para la realización de tareas, no sabiendo que tal condición no les imposibilita del todo obtener un empleo. Es decir, no existe un campo en la encuesta que indique la severidad de la discapacidad, pues si un individuo presenta una condición de discapacidad leve o moderada, tendría mayor oportunidad de incorporarse a un empleo remunerado, aspecto que se deja de lado en ambos Censos poblacionales, tanto en el año 2000 y 2010.

Por su parte, para el año 2010, la población total fue de 111.9 millones de habitantes, de las cuales 5.7 millones presentaron algún tipo de discapacidad (5.12\%), de esta cifra se derivan 2.97 millones de personas en el rango de edad entre 16 a 65 años, siendo 1.7 millones los disponibles para trabajar. Estadísticamente, este último aspecto evidencia puntualmente la discrepancia 
entre la población en edad de trabajar y las personas disponibles para trabajar, aproximadamente 1.27 millones de personas con discapacidad que se excluyen de la población disponible para trabajar, o se encuentran jubiladas, o se autoseleccionan como "imposibilitados para trabajar". De manera agregada, no considerar a todos estos individuos, o a un cierto porcentaje de ellos (excluyendo oportunamente a los que su severidad en la discapacidad les imposibilita trabajar), trae consigo sesgos en el diseño y eficiencia de políticas inclusivas de empleo.

Es importante destacar que la población con discapacidad aumentó de 2000 a 2010 en $96.15 \%$, las personas en esta condición en edad de trabajar en $61.79 \%$ y la población ocupada con discapacidad en $65.15 \%$. Una explicación de dicho cambio podría deberse a que las personas con discapacidad pudieron haber incrementado su nivel educativo y con ello la posibilidad de incorporarse al mercado de trabajo, dado que la falta de condiciones económicas suficientes, podrían estar presionando para que desarrollen alguna actividad económica.

Tabla 1

Población con y sin discapacidad en México (2000 y 2010)

\begin{tabular}{llccc}
\hline Concepto & 2000 & & 2010 & \\
& PSD & PCD & PSD & PCD \\
& & & & \\
\hline Población total & $92,993,303$ & $2,220,746$ & $105,402,663$ & $5,739,270$ \\
$\begin{array}{l}\text { Población en edad de } \\
\text { trabajar (16-65) }\end{array}$ & $55,177,665$ & $1,141,002$ & $66,982,735$ & $2,979,696$ \\
$\begin{array}{l}\text { Población disponible para } \\
\text { trabajar }{ }^{1}\end{array}$ & $54,591,454$ & $1,000,709$ & $49,941,712$ & $1,709,956$ \\
$\begin{array}{l}\text { Población económicamente } \\
\text { activa }\end{array}$ & $32,490,619$ & 438,121 & $41,116,100$ & $1,285,280$ \\
$\begin{array}{l}\text { Población ocupada } \\
\text { Población inactiva }\end{array}$ & $31,518,255$ & 415,812 & $38,943,011$ & $1,191,935$ \\
Población desempleada & $92,100,835$ & 562,588 & $8,825,612$ & 424,676 \\
& 972,364 & 22,309 & $2,173,089$ & 93,345
\end{tabular}

Fuente: Elaboración propia a partir de los Censos de Población y Vivienda de los años 2000 y 2010 . Nota: ${ }^{1}$ Se omite a los jubilados y auto reportados como imposibilitados para trabajar.

Con el motivo de justificar la elección de únicamente a los trabajadores en los empleos no calificados, en la tabla 2 se presentan los niveles de ocupación por tipo de empleo para ambos colectivos. Se observa que los trabajadores remunerados y asalariados se ubican con mayor frecuencia en empleos no calificados, que para personas sin discapacidad representan aproximadamente $85 \%$, mientras que para personas con discapacidad son más del $90 \%$, en ambos años. Para este segundo grupo, se incrementó la participación en empleos no 
calificados durante los diez años. Asimismo, los salarios promedio evidencian una considerable disparidad por tipo de empleo y por condición de salud.

Tabla 2

Niveles de ocupación por tipo de empleo de personas con y sin discapacidad (2000 y 2010)

\begin{tabular}{lllll}
\hline & 2000 & & 2010 & \\
& PSD & PCD & PSD & PCD \\
\hline Total de población ocupada & $31,518,255$ & 415,812 & $38,943,011$ & $1,191,935$ \\
Ocupados en empleos calificados & $4,476,413$ & 34,387 & $6,117,589$ & 93,543 \\
\% Ocupados en empleos calificados & $14.20 \%$ & $8.27 \%$ & $15.71 \%$ & $7.85 \%$ \\
Salario medio & 10,344 & 8,552 & 11,601 & 10,059 \\
Ocupados en empleos no calificados & $27,041,842$ & 381,425 & $32,825,422$ & $1,098,392$ \\
\% Ocupados en empleos no calificados & $85.80 \%$ & $91.73 \%$ & $84.29 \%$ & $92.15 \%$ \\
Salario medio & 3,591 & 2,935 & 4,819 & 3,992 \\
TOTAL & $100 \%$ & $100 \%$ & $100 \%$ & $100 \%$ \\
\hline
\end{tabular}

Fuente: Elaboración propia con base en los Censos de Población y Vivienda de los Años 2000 y 2010

Como muestra la evidencia descriptiva, los trabajadores con discapacidad ganan menos que los trabajadores sin discapacidad. Esto podría deberse a que tienen unas carácterísticas asociadas por sí mismas a un menor salario. Por ello, el objetivo de este trabajo se centra en determinar la existencia de una brecha salarial significativa entre los trabajadores con discapacidad y sin discapacidad para México, a su vez para saber en qué medida tal disparidad se explica por factores relacionados al capital humano, y qué cantidad no lo hace.

\subsection{Descomposición salarial}

Mediante la utilización de la metodología propuesta por FLF (2011), se obtienen, en términos logarítmicos y porcentuales, los componentes explicados y no explicados de la diferencia salarial entre ambos colectivos (trabajadores con y sin discapacidad). A su vez, y dado que la metodología lo permite, para la parte explicada se descompone el aporte (en porcentajes) de las variables personales, de tipo de contrato y de lugar de trabajo incluidas en las regresiones. En las tablas 3 y 4 se resumen la información de las estimaciones.

Primero, de acuerdo con la tabla 3, se observa que los trabajadores sin discapacidad en el año 2000, obtuvieron salarios mayores respecto a los individuos con discapacidad, entre 5.4 a $18.8 \%$ (la razón salarial), donde la desigualdad más grande se presentó en el decil más bajo. Es decir, para este año la tendencia indica que a menor salario, mayor disparidad salarial entre ambos colectivos.

Segundo, al observar la descomposición de la brecha entre los factores explicados y no explicados por las características, se obtiene que la sección no explicada tiene un aporte significativo sobre la disparidad a lo largo de toda la 
distribución, acentuándose en la cola derecha, donde su efecto llega a ser del $72.3 \%$, mientras que en la cola izquierda es del $44.4 \%$. Esto, aunado a lo anterior, permite refinar el análisis, pues para el decil más alto, a pesar de presentar la menor brecha salarial, ésta se debe en mayor medida a factores ajenos a la productividad. Es decir, a una latente discriminación. Por el lado opuesto de la distribución, aunque la brecha es comparativamente mayor al resto de percentiles, ésta se explica en su mayoría por las características incluidas en el modelo. Menores niveles de ingreso se asocian con mayor desigualdad. Sin embargo, para ésta, la mayor proporción es resultante de las características observadas, mientras que los niveles de salarios más altos, aunque con menor disparidad, presenta mayor discriminación, o al menos, no se explica por las características.

Estos resultados sugieren que en los colectivos cuyo ingreso salarial es superior, y que por defecto se asumen mejores características que permiten tales retribuciones, como mejor educación y experiencia, la discriminación asociada es mayor en este segmento poblacional, al igual que en el caso de los estudios por género, existe un "techo de cristal" que impide la igualdad en los salarios de los trabajadores, por el hecho de tener discapacidad sus retribuciones serán siempre comparativamente inferiores. Por el lado opuesto de la distribución, se presenta también el llamado efecto "piso pegajoso", que limita el crecimiento de los individuos cuyos rendimientos al salario son menores. En este caso, el hecho de tener discapacidad provoca que los ingresos permanezcan siempre por debajo de los trabajadores sin discapacidad. Este segmento poblacional es el de mayor marginación y vulnerabilidad, pues su bienestar de vida se ve condicionado por su condición de salud.

Otro aspecto a resaltar es que la sección no explicada retoma mayor relevancia en la brecha a partir del percentil 25, es decir, para la mayoría de los trabajadores con y sin discapacidad, la desigualdad en salarios se debe a factores ajenos a las características observadas. Esto, en materia de política laboral y de inclusión social es importante, pues la estrategia que en su momento debieron seguir los ejecutores de los programas públicos, se resume a resolver la discriminación asociada, poniendo especial interés en los deciles más elevados de ingreso.

Resulta interesante conocer que en los segmentos poblacionales cuyas características permiten mayor salario, es donde existió mayor discriminación. Este fenómeno que alude netamente a la ideología y la cultura de las personas, merma la calidad de vida de las personas con discapacidad, incluso en los grupos donde se asumiría que por mayor escolaridad, no debiera existir.

Ahora bien, la ventaja que tiene utilizar la medotodologia propuesta por FLF (2011) respecto a otras metodologías de descomposición, es que permite identificar el aporte que tiene cada una de las variables a la brecha salarial. En 
la parte inferior de la tabla 4, se agrupan en tres tipos de variables: i) personales (las que tienen que ver con las características de los individuos); ii) de tipo de contrato (horas de trabajo y formalidad); iii) de lugar de trabajo (referidas a la localidad y rama de actividad económica).

Al comparar los grupos de variables a lo largo de la distribución, se observa que, son las características de los individuos las que aportan en mayor medida al segmento explicado de la brecha. Por tanto, estos resultados otorga evidencia parcial de la teoría del capital humano, la cual indica que las características de los trabajadores condicionan el valor esperado de sus retribuciones. Los porcentajes asociados fluctúan entre 58.5, hasta un 99.6\%. este último referente al decil 90. Es decir, en este segmento poblacional la diversidad en las características personales de los trabajadores con y sin discapacidad son las que explican el $27.7 \%$ del diferencial salarial.

De todas las variables incluidas como personales, el gobierno solo tiene influencia sobre la escolaridad, pues es obligación del estado proveer una educación gratuita y de calidad. Un primer esfuerzo por mejorar las condiciones de vida de los trabajadores con discapacidad es proveerlos de educación, y con ello igualar las condiciones iniciales de competencia en el mercado laboral.

En segundo lugar, las variables del lugar de trabajo tienen una contribución entre 32.7 al $55.7 \%$. Aunque estas variables condicionan las retribuciones, la influencia que tienen las políticas públicas sobre estas es nula, pues, en definitiva, no es posible homogeneizar los salarios entre ramas de actividad económica, regiones o localidades rurales y urbanas, puesto que las condiciones y el costo de los factores productivos es distinto para cada uno. Sin embargo, lo que sí puede mejorarse es la contratación de individuos con discapacidad, y con ello incrementar las tasas de ocupación, para en un primer momento, insertarlos como población económicamente activa.

Y por último, resulta interesante observar las variables de tipo de contrato, pues, para la mayoría de los deciles estas tienen una influencia negativa. Es decir, propician la reducción de la brecha. Estos valores retoman importancia puesto que las horas de trabajo establecidas en los contratos laborales, y la condición de formalidad son aspectos que pueden fortalecerse a partir de la legislación que promueve la inclusión de personas con discapacidad a los centros de trabajo. Promover la constitución de organizaciones que incorporen personal con discapacidad puede incrementarse primeramente mediante estímulos fiscales, y segundo, para las personas con discapacidad conviene establecer un régimen rígido de horas de trabajo, pues, la flexibilización de trabajo a este colectivo no le es de beneficio. 
Tabla 3

Descomposición salarial en México entre trabajadores con y sin discapacidad en empleos no calificados (2000)

\begin{tabular}{llllll}
\hline Variable & \multicolumn{6}{l}{ Percentil en la distribución salarial } & \\
& $\mathbf{0 . 1 0}$ & $\mathbf{0 . 2 5}$ & $\mathbf{0 . 5 0}$ & $\mathbf{0 . 7 5}$ & $\mathbf{0 . 9 0}$ \\
\hline Log w/hora PSD & 0.783 & 0.965 & 1.136 & 1.317 & 1.519 \\
Log w/hora PCD & 0.659 & 0.862 & 1.053 & 1.236 & 1.441 \\
$\begin{array}{l}\text { Razón salarial } \\
\text { logarítmica }\end{array}$ & 1.188 & 1.119 & 1.079 & 1.066 & 1.054 \\
$\begin{array}{l}\text { Diferencia del Log } \\
\text { w/hora }\end{array}$ & 0.124 & 0.103 & 0.0823 & 0.0807 & 0.0775 \\
$\begin{array}{l}\text { Diferencia explicada } \\
\text { \% Explicado }\end{array}$ & 0.069 & 0.0435 & 0.0379 & 0.0348 & 0.0215 \\
$\begin{array}{l}\text { Diferencia no explicada } \\
\text { \% No explicado }\end{array}$ & 0.055 & 0.0599 & 0.0445 & 0.0459 & 0.056 \\
& $44.4 \%$ & $58.2 \%$ & $54.1 \%$ & $56.9 \%$ & $72.3 \%$
\end{tabular}

DESCOMPOSICIÓN DE LA SECCIÓN EXPLICADA ${ }^{3}$

\begin{tabular}{llllll}
\hline Variables personales & 0.0439 & 0.0258 & 0.0222 & 0.0250 & 0.0214 \\
$\begin{array}{l}\text { \% del diferencial } \\
\text { explicado }\end{array}$ & $63.7 \%$ & $59.2 \%$ & $58.5 \%$ & $71.9 \%$ & $99.6 \%$ \\
$\begin{array}{l}\text { Variables de contrato } \\
\text { \% del diferencial }\end{array}$ & 0.0026 & -0.0002 & -0.0017 & -0.0059 & -0.0119 \\
$\begin{array}{l}\text { explicado } \\
\begin{array}{l}\text { Variables lugar de } \\
\text { trabajo }\end{array}\end{array}$ & $3.7 \%$ & $-0.4 \%$ & $-4.6 \%$ & $-16.8 \%$ & $-55.2 \%$ \\
$\begin{array}{l}\text { \% del diferencial } \\
\text { explicado }\end{array}$ & $32.7 \%$ & $41.3 \%$ & $47.0 \%$ & $46.8 \%$ & $55.7 \%$ \\
\hline
\end{tabular}

Fuente: Elaboración propia. Nota: Tamaño muestral $=1,706,964$ observaciones ${ }^{3}$ a) variables personales: interacción, sexo, rangos de edad, nivel educativo, experiencia, experiencia al cuadrado y pertenencia étnica; b) variables de tipo de contrato: horas de trabajo y formalidad; c) variables de lugar de trabajo: tipo de localidad urbana, rama de actividad económica y región.

Una vez descrita la situación del año 2000, conviene ahora realizar un análisis similar una década más tarde. Observando la tabla 5, para el año 2010, la situación salarial mejoró (en términos reales) para ambos grupos de trabajadores, lo cual trajo consigo una ligera disminución de la brecha para todos los deciles de ingreso, siendo en la cola izquierda donde se presentó la mayor reducción, absoluta y proporcionalmente. En este año, el salario de los trabajadores sin discapacidad fue mayor entre el 4 al 7.2\%, respecto a los trabajadores con discapacidad ( la razón salarial), siendo nuevamente en el decil más bajo donde se presentó la mayor disparidad. 
Sin embargo, para el 2010, a diferencia del 2000, no se observa una clara tendencia a la reducción de la desigualdad a medida que incrementa el salario de los trabajadores, pues, la menor disparidad se presenta en la mediana de la distribución. La brecha más amplia se observa en el decil inferior, posteriormente se reduce para el decil intermedio, y después se revierte tal tendencia, y se amplía nuevamente para el último decil.

Un primer esfuerzo por explicar esto se asocia directamente con las características de los trabajadores con discapacidad, ya que la mejora en los niveles de educación resultó en incrementos salariales para los segmentos intermedios de la distribución.

Ahora bien, observando los porcentajes asociados a la sección explicada y no explicada de la brecha, resulta nuevamente que en el decil más bajo, los factores relacionados con las características son los que contribuyen a explicar en mayor medida la desigualdad en los salarios, mientras que para el decil más alto, lo que se observó una década anterior, continúa vigente, la discriminación asociada es mayor a la sección explicada. Sin embargo, no es este decil donde se presenta la mayor "discriminación", ya que es en el decil 75 donde ocurre que el $80.5 \%$ de la brecha es atribuida a factores ajenos a productividad.

A lo largo de la distribución son en los deciles intermedios donde la sección no explicada retoma mayor importancia, y es precisamente en ésta donde es menor la desigualdad. Con esta información, aunada a la del año 2000, podría evidenciarse cierta correlación, pues en los segmentos de ingreso donde la desigualdad es menor, la discriminación es la que explica en su mayoría la brecha.

Analizando la descomposición acorde a los grupos de variables personales, de contrato y de lugar de trabajo, para este año, se presentan resultados similares a los del año 2000. Las características individuales son las que aportan un mayor porcentaje a la sección explicada de la brecha, incluso siendo más de cien por ciento en la cola derecha de la distribución, lo cual indica que en este segmento, la diferencia explicada se debe netamente a las características personales.

Estas cifras dan validez parcial de la teoría del capital humano, la cual postula que las disparidades salariales están en función de las características de educación, sexo, edad y experiencia de los individuos. Sin embargo, mediante la descomposición se obtiene que, aunque la mayor proporción de la sección explicada es atribuida a variables personales, para la mayoría de los deciles, este segmento explicado es menor a comparación de la diferencia no explicada por las características. Esto plantea nuevos retos teóricos, pues empíricamente 
se está demostrando que son otros factores los que influyen sobre el nivel de salarios que obtienen los trabajadores.

Por otro lado, las variables relacionadas con el lugar de trabajo pierden importancia sobre la parte explicada, esto con respecto al año 2000. Esto podría explicarse por los avances en materia de inclusión que se han logrado a lo largo de la década, entre ramas de actividad económica y regiones. Sin embargo, estas variables afectarán siempre significativamente a la brecha, pues los costos de vida son diferentes.

Finalmente, las variables relacionadas con el tipo de contrato, nuevamente presentaron el signo negativo, lo cual indica que reducen la brecha de salarios entre ambos colectivos, aunque pierden considerablemente aporte proporcional respecto al año 2000.

Analizados ambos años de manera separada, y puesto que el tamaño muestral lo permite ya que captura la representatividad de ambos colectivos, es oportuno analizar los cambios sucedidos a lo largo de la década, al menos de los niveles de salario y la brecha. Para ello, la tabla 5, presenta los cambios porcentuales sucedidos en el periodo 2000-2010.

En primer lugar, se obtienen las tasas de crecimiento del logaritmo del salario por hora para los trabajadores sin y con discapacidad, se observa que el mayor incremento se presentó en el decil más bajo de la distribución, 21.7 y 34.9\%, respectivamente. Podría afirmarse que las políticas de mejora salarial en los empleos no calificados surtieron mayor efecto en este segmento poblacional. Aunado a esto, se observa que la brecha disminuyó en $48.7 \%$, en este decil, la lucha contra la desigualdad salarial fue comparativamente mayor.

En el extremo opuesto de la distribución, se obtuvo un crecimiento salarial de 3.7 y $5 \%$ para trabajadores sin y con discapacidad, respectivamente. Por su parte, la brecha presentó una reducción del $20.8 \%$. Con lo anterior, se puede concluir que, a lo largo de los diez años, el salario de los individuos con discapacidad mejoró en demasía, lo cual contribuyó a acortar las brechas.

Resumiendo toda la información anterior, y enfrentándola con la hipótesis planteada al inicio de esta investigación, en primera instancia, es evidente que la desigualdad salarial entre ambos colectivos está presente para ambos años, y a su vez, esta disminuyó durante la década de referencia, siendo en el decil más bajo donde la mejora fue comparativamente mayor. 
Tabla 4

Descomposición salarial nacional entre trabajadores con y sin discapacidad en empleos no calificados (2010)

\begin{tabular}{|c|c|c|c|c|c|}
\hline \multirow[t]{2}{*}{ Variable } & \multicolumn{5}{|c|}{ Percentil en la distribución salarial } \\
\hline & 0.10 & 0.25 & 0.50 & 0.75 & 0.90 \\
\hline Log w/hora PSD & 0.953 & 1.104 & 1.233 & 1.402 & 1.575 \\
\hline Log w/hora PCD & 0.889 & 1.048 & 1.186 & 1.346 & 1.513 \\
\hline $\begin{array}{l}\text { Razón salarial } \\
\text { logarítmica }\end{array}$ & 1.072 & 1.053 & 1.040 & 1.042 & 1.041 \\
\hline $\begin{array}{l}\text { Diferencia del Log } \\
\text { w/hora }\end{array}$ & 0.0636 & 0.0558 & 0.0476 & 0.0568 & 0.0614 \\
\hline Diferencia explicada & 0.04 & 0.0126 & 0.0137 & 0.0111 & 0.0215 \\
\hline \% Explicado & $62.89 \%$ & $22.58 \%$ & $28.78 \%$ & $19.54 \%$ & $35.02 \%$ \\
\hline $\begin{array}{l}\text { Diferencia no } \\
\text { explicada }\end{array}$ & 0.0236 & 0.0431 & 0.0339 & 0.0457 & 0.0399 \\
\hline$\%$ No explicado & $37.1 \%$ & $77.2 \%$ & $71.2 \%$ & $80.5 \%$ & $65.0 \%$ \\
\hline \multicolumn{6}{|c|}{ DESCOMPOSICIÓN DE LA SECCIÓN EXPLICADA ${ }^{4}$} \\
\hline Variables personales & 0.0349 & 0.0117 & 0.0124 & 0.0110 & 0.0244 \\
\hline $\begin{array}{l}\% \text { del diferencial } \\
\text { explicado }\end{array}$ & $87.1 \%$ & $93.1 \%$ & $90.6 \%$ & $98.7 \%$ & $113.3 \%$ \\
\hline Variables de contrato & 0.0009 & -0.0006 & -0.0012 & -0.0014 & -0.0035 \\
\hline $\begin{array}{l}\% \text { del diferencial } \\
\text { explicado }\end{array}$ & $2.2 \%$ & $-4.5 \%$ & $-9.1 \%$ & $-13.0 \%$ & $-16.2 \%$ \\
\hline $\begin{array}{l}\text { Variables lugar de } \\
\text { trabajo }\end{array}$ & 0.0046 & 0.0014 & 0.0025 & 0.0016 & 0.0007 \\
\hline $\begin{array}{l}\% \text { del diferencial } \\
\text { explicado }\end{array}$ & $11.4 \%$ & $11.5 \%$ & $18.1 \%$ & $14.8 \%$ & $3.3 \%$ \\
\hline
\end{tabular}

Fuente: Elaboración propia. Nota: Tamaño muestral $=1,478,259$ observaciones. ${ }^{4}$ a) variables personales: interacción, sexo, rangos de edad, nivel educativo, experiencia, experiencia al cuadrado y pertenencia étnica; b) variables de tipo de contrato: horas de trabajo y formalidad; c) variables de lugar de trabajo: tipo de localidad urbana, rama de actividad económica y región.

A su vez, en los deciles intermedios de la distribución se presentó un incremento en la proporción de la disparidad debida a factores no explicados por productividad. Es decir, la discriminación asociada cobró mayor importancia en estos segmentos poblacionales. Por el contrario, en los extremos de la distribución, la sección no explicada perdió relevancia sobre la brecha. Este aspecto contradice parcialmente la hipótesis, pues se esperaba que en los extremos, la parte atribuida a discriminación fuera mayor respecto a la explicada a las características. 
Tabla 5

Cambios porcentuales en la década 2000 - 2010, de salarios y la brecha salarial entre trabajadores con y sin discapacidad, en empleos no calificados

\begin{tabular}{llllll}
\hline Variable & \multicolumn{2}{l}{ Percentil en la distribución salarial } \\
& $\mathbf{0 . 1 0}$ & $\mathbf{0 . 2 5}$ & $\mathbf{0 . 5 0}$ & $\mathbf{0 . 7 5}$ & $\mathbf{0 . 9 0}$ \\
\hline Log w/hora PSD & $21.7 \%$ & $14.4 \%$ & $8.5 \%$ & $6.5 \%$ & $3.7 \%$ \\
Log w/hora PCD & $34.9 \%$ & $21.6 \%$ & $12.6 \%$ & $8.9 \%$ & $5.0 \%$ \\
$\begin{array}{l}\text { Diferencia del } \\
\text { w/hora }\end{array}$ & $-48.7 \%$ & $-45.8 \%$ & $-42.2 \%$ & $-29.6 \%$ & $-20.8 \%$ \\
\hline
\end{tabular}

Fuente: elaboración propia con base en las tablas 3 y 4.

Es relevante mencionar que dentro de la sección efectivamente explicada por las características observadas, para todos los deciles, son las variables incluidas como personales las que propician la disparidad en los salarios. Es decir, la teoría del capital humano está vigente de manera parcial, pues, los factores no relacionados con esta tienen un peso mayor sobre la desigualdad.

Si se comparan estos resultados con los obtenidos en diversas investigaciones, se encuentra convergencia en que la discriminación es un fenómeno que continúa presente al momento de las asignaciones salariales. Con el estudio elaborado por Márquez y Reyes (2014), con datos de la ENIGH del 2010, al utilizar el emparejamiento de Nopo, el porcentaje de la brecha asociado a factores no explicados es del $68 \%$, el cual se asemeja a los resultados de este documento, en donde para el mismo año 2010 este porcentaje fluctúa entre 43.3 y $66.8 \%$.

Por su parte, la investigación realizada para Coahuila de Castro, Rodríguez y Galván (2017) evidencia un nivel de discriminación asociado de $90 \%$ en el 2000 , y de $60 \%$ en el 2010. A pesar que en el primer año, el valor estimado dista mucho de lo evidenciado en esta investigación para todo el país, el segundo, sí se enmarca dentro del intervalo de discriminación encontrado para la distribución salarial.

Ahora bien, cotejando con la evidencia internacional, se demuestra que los niveles de discriminación son similares a los observados en Francia (Halima y Rococo, 2014), y España (Pagán y Marchante, 2004), mientras que son mayores comparados con los existentes en Perú (Barletti y Yllesca, 2014). Se podría encontrar en este punto, que este fenómeno se agudiza en las naciones con mayor nivel de desarrollo, sin embargo, se requiere realizar un estudio más exhaustivo abarcando mayor cantidad de países, para comprobar esta hipótesis. En sintensis, la desigualdad salarial entre personas con y sin discapacidad es una realidad que continúa latente. Evidenciado empíricamente en esta investigación, la disparidad se explica en mayor medida por características no 
relacionadas con la productividad, por lo que la muy probable discriminación a la que se enfrenta el colectivo de personas con discapacidad, merma su calidad de vida y las aspiraciones de movilidad social. Sin embargo, es importante mencionar que la discapacidad puede estar relacionada, con una menor productividad, al menos en función del tipo de ocupación que se desempeña y de si hay o no adaptación de las tareas que realizan con el tipo de discapacidad de los individuos.

Malo y Pagán (2012) en su estudio separan a las personas con discapacidad que no experimentan limitaciones (por tenerla compensada con alguna ayuda técnica o personal, o por la levedad de su discapacidad). Al no experimentar limitaciones, se puede inferir que su discapacidad no reduce la productividad $\mathrm{y}$, de hecho, para este grupo las diferencias salariales responden casi en exclusiva a las características (baja educación y formación de una manera muy importante) y muy poco o nada a discriminación. Ahora bien, para las personas con una discapacidad que genera limitaciones ambos factores (características y potencial discriminación) contribuyen a explicar las diferencias salariales, pero para este grupo no se puede estar seguro de que la propia discapacidad genere menor productividad. Por lo que, desde esta perspectiva, es importante seguir indagando sobre el tema.

Por otra parte, los retos en materia de política pública son múltiples, y simultáneos, pues en primer lugar, para mejorar la inclusión social de personas con discapacidad se deben fortalecer los mecanismos que promueven el empleo de este colectivo. Incrementar la tasa de participación debe ser un punto a resolver dentro de la agenda pública, con ello, es posible mejorar sus condiciones de vida al garantizar un ingreso salarial que permita la satisfacción de sus necesidades, y al mismo tiempo, reducir la carga del estado hacia la atención de este grupo vulnerable.

Segundo, mejorar la igualdad de condiciones iniciales entre personas con y sin discapacidad, haciendo énfasis en la educación y en los términos de contratación (formalidad en el empleo y jornadas laborales). Este par de elementos propician la reducción de las brechas, ya que la educación promueve la mejora en los rendimientos salariales mientas que la adecuación de los contratos garantiza un empleo digno.

Tercero, la lucha contra la discriminación se debe propiciar de manera simultánea a los dos puntos anteriores, y para todos los rangos de ingreso, pues es un fenómeno presente en todos los niveles socioeconómicos. Se propone que la colaboración sea de manera horizontal entre gobierno, organizaciones de la sociedad civil y empresas, pues estos aspectos culturales negativos de la sociedad mexicana limitan el progreso. Como se demuestra con los resultados, 
el "piso pegajoso" y el "techo de cristal" también son fenómenos que afectan a los individuos con discapacidad.

\section{Conclusiones}

Una vez desarrollada la estadística descriptiva de los censos en relación a los niveles de ocupación, así como la descomposición salarial, se vuelve necesario comprobar y dar respuesta a cada una de las preguntas de investigación planteadas al inicio de esta investigación. En este sentido, el objetivo general de esta investigación, referido a la comprobación empírica de la desigualdad salarial entre trabajadores con y sin discapacidad se cumple, pues, primero, con los datos presentados se evidencia que, en efecto, existe una brecha salarial entre trabajadores con y sin discapacidad de 5.4 a $18.8 \%$ para el año 2000 , y entre el 4.1 a $7.2 \%$ para el 2010 . Por tanto, se puede afirmar que la discriminación se agudizó en los segmentos poblacionales con salarios intermedios, en donde se ubica la mayoría de los trabajadores.

Finalmente, puesto que el documento centra su atención en demostrar la validez de la hipótesis, es oportuno escribirla nuevamente: "En ambos años, 2000 y 2010, existe desigualdad salarial entre personas con y sin discapacidad, y a lo largo de la década, sobre la brecha existente en los extremos de la distribución salarial, tienen mayor participación los factores ajenos a productividad"

Primero, en efecto, la desigualdad y discriminación salarial entre personas con y sin discapacidad son fenómenos que continúan latentes, al menos en los empleos no calificados. Segundo, a lo largo de la década se demuestra que la desigualdad es menor, sin embargo, los factores no asociados con productividad continúan contribuyendo en gran medida a la brecha salarial. Y tercero, para ambos extremos de la distribución no ocurre que la discriminación (el porcentaje asociado a los factores no explicados) sea mayor que el porcentaje explicado, sí lo hace para el decíl más elevado, pero para el decil más bajo no.

Los datos presentados en este artículo revelan que el fenómeno de discriminación se encuentra latente en el mercado laboral mexicano, y que hasta cierto punto, no han sido eficientes las políticas de inclusión social y de empleo para este colectivo. Es necesario entonces, reevaluar y plantear con claridad cuáles son las estrategias que se deben seguir para mitigar la discriminación, aspecto que se relaciona completamente con las acciones a realizar en pro de los derechos humanos.

Conociendo de ante mano la existencia de desigualdades entre estos colectivos, la inclusión de las personas con discapacidad al mercado laboral constituye un 
reto actual y vigente para los tomadores de decisiones en materia de política pública y laboral. Los programas establecidos en el ámbito federal y estatal deben ser evaluados para demostrar su eficiencia y eficacia, y por último, las medidas nuevas a implementar deben construirse a partir del consenso con personas con discapacidad, pues son ellos quienes conocen en mayor medida las condiciones de vida de este colectivo, sus necesidades y oportunidades de participación y desarrollo.

Es importante mencionar que como una futura línea de investigación se podría retomar el estudio y análizar la severidad de la discapacidad, a traves de una variable que indique la discapacidad multiple, al considerar que los individuos tienen distintos tipos de limitaciones físicas, ya que esta puede tener un efecto diferente en la productividad y por lo tanto en la desigualdad y en la discriminación de los individuos con y sin discapacidad.

\section{Referencias}

[1] Baldwin, M. (1994) "Estimating Wage Discrimination against Workers with Disabilities". Cornell Journal of Law and Public Policy, 3(2), 276-290.

[2] Baldwin, M, y Johnson, W. (1995). "Labor Market Discrimination against Women with Disabilities". Industrial Relations. A Journal of Economy and Society, 34(4), 555-577. https://doi.org/10.1111/j.1468-232X.1995.tb00388.x

[3] Barletti, S, y Yllesca, S. (2014). "Brechas salariales como resultado de la discriminación de discapacitados en el Perú urbano 2012: ¿Existen realmente?" Lima, Perú: Universidad del Pacifico. Working Paper Universidad del Pacifico; Investigación Económica II; Semestre 2014-II

[4] Becker, G. (1957). The economics of discrimination. University of Chicago Press.

[5] Berman, B. R. (1974). "Occupational segregation, wages and profits when employers discriminate by race or sex". Eastern Economic Journal. Eastern Economic Association. 1(2), 103-110. https://doi.org/10.1007/s10734-0053864-3

[6] Benito, S, Glassman, T. y Hiedemann, B. (2016). "Disability and labor market earnings. Hearing earnings gaps in the United States". Journal of Disability Policy Studies. 27(3) 178-188. https://doi.org/10.1177/1044207316658752

[7] Blinder, A. S. (1973). "Wage Discrimination: Reduced Form and Structural Estimates". The Journal of Human Resources. 8(4) 436-455. https://doi.org/10.2307/144855

[8] Castro, D, Rodríguez R, y Galván. K. (2017). Brecha salarial y discriminación por discapacidad en Coahuila del 2000 al 2010. En. Ochoa, G. y Torres, A. (Ed). Los retos del cambio económico actual: revisión y aplicaciones para el caso mexicano. 49-73. Monterrey, Nuevo León. Ediciones de Laurel.

[9] Aigner, D. J and Cain, Glen G. C. (1977). "Statistical theories of discrimination in labor markets". ILR Review. 30(2). 175-187. https://doi.org/10.2307/2522871

[10] CE, Comision Europea. (2014). "Cómo combatir la brecha salarial entre hombres y mujeres en la Unión Europea”. Bélgica: Oficina de Publicaciones de la Unión Europea. 
[11] Fairlie, R. W. (1999). "The Absence of the African-American Owned Business: An Analysis of the Dynamics of Self-Employment". Journal of Labor Economics. 17(1), 80-108. https://doi.org/10.1086/209914

[12] Fortin, N., Lemieux, T. y Firpo, S. (2011). "Decomposition Methods in Economics", Handbook of Labor Economics, 4(1), 1-102.

[13] Baquero, J, Guataquí, J. C. y Sarmiento, L. (2000). "Un marco analítico de la discriminación laboral". Borradores de investigación, 8, 1-31.

[14] Halima, B, Mohamed Ali y Rococo, E. (2014). "Wage differences according to health status in France". Social Science \& Medicine, 120,260-268. DOI: 10.1016/j.socscimed.2014.09.035

[15] Heckman, J, J. (1979). "Sample Selection Bias as a Specification Error". Econometrica. 47(1), 153-161. https://doi.org/10.2307/1912352

[16] INEGI, Instituto Nacional de Estadística y Geografía. (2011). “Clasificadores del Censo de Población y Vivienda 2010". Aguascalientes, México. INEGI.

[17] Jones, M, Latreille, P, y Slone, P. (2006). "Disability, gender and the labour market". Oxford Economic Papers. 58, 407-449. doi:10.1093/oep/gpl004

[18] Kidd, M, P., Sloane, P y Ferko, I. (2000). "Disability and the labour market: an analysis of British males". Journal of Health Economics. 19(6),961-981. https://doi.org/10.1016/S0167-6296(00)00043-6

[19] Lago, I. (2002). "La discriminación salarial por razones de género: un análisis empírico para el sector privado en España". Revista Española de Investigaciones Sociológicas, 98, 171-196.

[20] Lanot, G y Walker. L. (1998). "The union/non-union wage differential: an application of semi-parametric". Journal of Econometrics, 84 (2), 327 -349.

[21] Malo, M. A. (2007). "La definición de la discapacidad en la investigación económica: Una reflexión necesaria sobre qué características debería cumplir". Estudios de Economía Aplicada. 25 (2) 407-428.

[22] Malo, M y Pagan R. (2012). "Wage differentials and disability across Europe: Discrimination and/or lower productivity?" International Labour Review. 151(1-2), 43-60.

[23] Malo, M. A. (2017). "Discriminación salarial y discapacidad: los datos de política de empleo". Revista Panorama Social. 26, 69-78.

[24] Márquez, C, y Reyes. J. (2015). “Análisis de la brecha de ingresos entre las personas con y sin discapacidad en ocupaciones no calificadas". Revista Latinoamericana de Población, 16, 33-58.

[25] Mitra, S, y Sambamoorthi, U. (2008). "Disability and the Rural Labor Market in India: Evidence for Males in Tamil Nadu”. World Development. 36(5), 934 - 952. https://doi.org/10.1016/j.worlddev.2007.04.022

[26] Murillo, I, y Hipólito, S. (2013). "La Gran Recesión y el diferencial salarial por género en España”. Hacienda Pública Española / Review of Public Economics, 208(1), 39-76. DOI: 10.7866/HPE-RPE.14.1.2

[27] Phelps, E. (1972). "The statistical theory of racism and sexism". The American Economic Review. 62.(4), 659-661. https://www.jstor.org/stable/1806107

[28] Nopo, H. (2004). "Matching as a Tool to Decompose Wage Gaps". Discussion Paper Series. 981, 1-48.

[29] Oaxaca, R. (1973). "Male-Female wage differentials in urban labor markets". International Economic Review. 4(3), 693-709. https://doi.org/10.2307/2525981 
[30] OMS, Organización Mundial de la Salud. (2001). “Clasificación Internacional del Funcionamiento, de la Discapacidad y de la Salud". Editado por Ministerio de Trabajo y Asuntos Sociales. Secretaría General de Asuntos Sociales. Instituto de Migraciones y Servicios Sociales. Madrid: Centro Colaborador en España junto con la RHHD y OMS/OPS.

[31] OMS, Organización Mundial de la Salud, y Banco Mundial BM. (2011). "Informe Mundial sobre la Discapacidad”. Ginebra, Suiza: Ediciones de la OMS.

[32] ONU, Organización de las Naciones Unidas. (2008). "Convención sobre los derechos de las personas con discapacidad”. Nueva York: Organización de las Naciones Unidas.

[33] Pagán, R, y Marchante, A. (2004). “Análisis de las diferencias salariales por discapacidad en España: el caso de los varones”. Hacienda Pública Española. Revista de Economía Pública, 171(4), 75-100.

[34] Tenjo, J, Ribero, R y Bernat, L. (2005). "Evolución de las diferencias salariales por sexo en seis países de América Latina: un intento de interpretación" Documentos CEDE (Universidad de los Andes), 18, 1-59.

[35] Skogman, P. (2004). "Occupational attainment and earnings: The case of the disabled”. Labour. 18(3), 262-278. https://doi.org/10.1111/j.11217081.2004.00276.x

[36] Yunker, H. (1988). "Perceptions of severely and multiply disabled persons. Journal of Multi-handicapped Person. 1(1), 1-12. 